\title{
Explicit constructions of triple systems for Ramsey-Turán problems
}

\author{
Dhruv Mubayi * Vera T. Sós ${ }^{\dagger}$
}

February 17, 2007

\begin{abstract}
We explicitly construct four infinite families of irreducible triple systems with Ramsey-Turán density less than the Turán density. Two of our families generalize isolated examples of Sidorenko [14], and the first author and Rödl [12]. Our constructions also yield two infinite families of irreducible triple systems whose Ramsey-Turán densities are exactly determined.
\end{abstract}

For an $r$-graph $\mathcal{F}$, the Turán number $\operatorname{ex}(n, \mathcal{F})$ is the maximum number of edges in an $n$ vertex $r$-graph containing no copy of $\mathcal{F}$. It is well known that $\pi(\mathcal{F})=\lim _{n \rightarrow \infty} \operatorname{ex}(n, \mathcal{F}) /\left(\begin{array}{l}n \\ r\end{array}\right)$ exists, but these numbers are very hard to determine when $r \geq 3$. For example, until very recently [10] no nontrivial infinite family $\left\{\mathcal{F}_{i}\right\}$ of triple systems has been constructed for which $\pi\left(\mathcal{F}_{i}\right)$ is known (by "nontrivial" we mean that for every $\mathcal{F}_{i}$, there are no two vertices $x, y$ of $\mathcal{F}_{i}$ for which (1) no edge contains both $x$ and $y$, and (2) $x u v$ is an edge iff $y u v$ is an edge). Two examples that are known, and used in this note, are $\pi\left(F_{5}\right)=2 / 9$, and $\pi(F(3,2))=4 / 9$, where $F_{5}=\{123,124,345\}$ and $F(3,2)=\{123,124,125,345\}$.

Many of the (conjectured) extremal examples for (hyper)graph Turán problems have large independent sets. Motivated by this observation, Erdős and Sós imposed a restriction on the

${ }^{*}$ Department of Mathematics, Statistics, and Computer Science, University of Illinois, 851 S. Morgan Street, Chicago, IL 60607-7045, email: mubayi@math.uic.edu; research supported in part by the National Science Foundation under grants DMS-9970325, DMS-0400812, and an Alfred P. Sloan fellowship

${ }^{\dagger}$ Alfred Rényi Institute of Mathematics, Hungarian Academy of Sciences, POB 127 Budapest H-1364, Hungary, email: sos@renyi.hu; research supported in part by Hungarian OTKA grants T-042750 and T-038210

1991 Mathematics Subject Classification: 05C35, 05C65, 05D05

Keywords: Ramsey Turán problems, extremal hypergraph theory 
underlying $r$-graphs in this problem, namely that they should not have large independent sets. This new class of problems has become known as the Ramsey-Turán problems. More precisely, for $0<\delta \leq 1$,

$$
\operatorname{ex}(n, \mathcal{F}, \delta)=\max \{|\mathcal{G}|: \mathcal{G} \text { is an } r \text {-graph with } \mathcal{F} \not \subset \mathcal{G} \text { and } \alpha(\mathcal{G})<\delta n\}
$$

or zero if no such hypergraph exists. The Ramsey-Turán number $\rho(\mathcal{F})$ is defined as

$$
\sup _{\delta(n)}\left\{\limsup _{n \rightarrow \infty} \frac{\operatorname{ex}(n, \mathcal{F}, \delta(n))}{\left(\begin{array}{l}
n \\
r
\end{array}\right)}: \delta(n) \rightarrow 0 \text { as } n \rightarrow \infty\right\} .
$$

Since obviously $\rho(\mathcal{F}) \leq \pi(\mathcal{F})$ for every $\mathcal{F}$, a fundamental question is whether equality holds. A sequence of papers $[1,3,16]$ showed that in the case that $\mathcal{F}$ is a complete (2-uniform) graph, $\rho(\mathcal{F})<\pi(\mathcal{F})$. It was therefore a surprise when Erdős and Sós [4] proved that for $r$-graphs when $r \geq 3$, this does not hold. Call an $r$-graph $\mathcal{H}$ locally dense if for every edge $E \in \mathcal{H}$, there is another edge $E^{\prime} \in \mathcal{H}$ with $\left|E \cap E^{\prime}\right| \geq 2$.

Theorem 1. (Erdős-Sós [4]) Let $r \geq 3$ and $\mathcal{H}$ be a locally dense $r$-graph. Then $\rho(\mathcal{H})=\pi(\mathcal{H})$.

On the other hand, it is proved in [4] that $r$-graphs $\mathcal{F}$ exist $(r \geq 3)$ for which $0=\rho(\mathcal{F})<\pi(\mathcal{F})$. Motivated by these examples, Erdős and Sós asked whether there exist $r$-graphs $(r>2)$ with

$$
0<\rho(\mathcal{F})<\pi(\mathcal{F})
$$

This was answered positively by Frankl and Rödl [8] for every $r>2$, who showed that there exist infinitely many $r$-graphs for which (1) holds however, they did not obtain a single explicit example. Subsequently, Sidorenko [14], using ideas from [8] proved that for the 3-graph $F_{7}=$ $\{123,145,167,245,267,345,367,467,567\}$, inequality (1) holds. Recently, the first author and Rödl [12] proved that $F(3,3)=\{124,125,126,134,135,136,234,235,236,456\}$ is another example.

Call an $r$-graph $\mathcal{H}$ reducible if

1) it is disconnected, or

2) its vertex set can be partitioned into $X \cup Y$, such that no edges of $\mathcal{H}$ are contained in $Y$, and all edges $E$ of $\mathcal{H}$ with $E \cap X$ and $E \cap Y$ both nonempty have the form $\left\{x, y_{1}, \ldots, y_{r-1}\right\}$, where $x \in X$ is fixed, $\left\{y_{1}, \ldots, y_{r}\right\} \subset Y$, and $\left\{\left\{y_{1}, \ldots, y_{r-1}\right\}:\left\{x, y_{1}, \ldots, y_{r-1}\right\} \in \mathcal{H}\right\}$ is $(r-1)$-partite.

If $\mathcal{H}$ is not reducible, then $\mathcal{H}$ is irreducible. It follows [13] from the definition of $\rho$ that for every reducible $\mathcal{H}$, there is an irreducible $\mathcal{H}^{\prime} \subset \mathcal{H}$ for which $\rho\left(\mathcal{H}^{\prime}\right)=\rho(\mathcal{H})$ (in Case $1, \mathcal{H}^{\prime}$ is an 
appropriately chosen component, and in Case $2, \mathcal{H}^{\prime}=\mathcal{H}-Y$ ). Therefore, it makes sense to ask for $\rho(\mathcal{H})$ only when $\mathcal{H}$ is irreducible.

In this note, we use essentially the same ideas from [8] to explicitly construct four infinite families of irreducible triple systems for which $0<\rho<\pi$. Our constructions are in the spirit of [14], but we obtain more variety (in particular, infinite families) with no extra effort. Although the underlying principle behind our construction is a rather general phenomenon (see (3)), our lack of understanding of $\pi$ limits our approach.

One of our families (see Example 1) contains $F(3,3)$, and another (see Example 3 ) contains $F_{7}$. Thus our contribution can be viewed as a generalization of results in $[12,14]$. Our constructions also yield two infinite families of irreducible triple systems $\mathcal{F}_{i}$ for which $\rho\left(\mathcal{F}_{i}\right)$ is determined. The values in the two cases are 2/9 and 4/9 (see Examples 2 and 3). Thus in this sense, our understanding of $\rho$ for hypergraphs is greater than that for $\pi$ (we can think of the notion of irreducible for $\rho$ as analogous to the notion of nontrivial for $\pi$ ).

Given an $r$-graph $\mathcal{H}$, let $\mathcal{H}^{*}$ be an $r$-graph obtained from $\mathcal{H}$ by replacing a vertex $v$ with $r$ vertices $v_{1}, \ldots, v_{r}$, replacing each edge $E$ containing $v$ with $r$ edges $E_{1}, \ldots, E_{r}$, where $E_{i}=E-v \cup\left\{v_{i}\right\}$, and adding the edge $\left\{v_{1}, \ldots, v_{r}\right\}$.

The main tool for the constructions is the following theorem. Although we proved it independently, later we noticed that the main part of it is proved in ([8] Lemma 2.3).

Theorem 2. Let $\mathcal{H}$ be an $r$-graph, and $\mathcal{H}^{*}$ be obtained from $\mathcal{H}$ by replacing any vertex $v$. Then $\rho\left(\mathcal{H}^{*}\right) \leq \pi(\mathcal{H})$. If, in addition, $\mathcal{H}$ is locally dense, then $\rho\left(\mathcal{H}^{*}\right)=\pi(\mathcal{H})$.

Proof. The first part is proved in [8]. For the last statement, Theorem 1 yields $\rho\left(\mathcal{H}^{*}\right) \leq \pi(\mathcal{H})=$ $\rho(\mathcal{H})$. Since $\mathcal{H} \subset \mathcal{H}^{*}$, the result follows.

A vertex multiplication in a hypergraph $\mathcal{H}$ is the replacement of a vertex $v$ in $\mathcal{H}$ by a finite set of vertices $\left\{v_{1}, \ldots, v_{k}\right\}$, and the replacement of every edge $E$ containing $v$ by the $k$ edges $E-v \cup\left\{v_{i}\right\}$. If $\mathcal{H}^{\prime}$ is obtained from $\mathcal{H}$ by a finite sequence of vertex multiplications, then we say that $\mathcal{H}^{\prime}$ is a blowup of $\mathcal{H}$. It is easy to see that if $\mathcal{H}$ is locally dense, then $\mathcal{H}^{\prime}$ is locally dense as well. Also, it is well-known (see, e.g. [15]) that

$$
\pi(\mathcal{H})=\pi\left(\mathcal{H}^{\prime}\right)
$$

Note that if $\mathcal{F}^{*}$ is obtained from $\mathcal{F}$ by replacing a non-isolated vertex of $\mathcal{F}$, then $\mathcal{F}^{*}$ contains the hypergraph $F(3,2)=\{567,467,367,345\}$, and it is known [11] that $\pi(F(3,2)) \geq 4 / 9$. Therefore 
$\pi\left(\mathcal{F}^{*}\right) \geq 4 / 9$

Our constructions below yield infinite families of irreducible 3-graphs, since in each case (except Example 2) we begin with an arbitrary blowup $\mathcal{F}$ of an irreducible 3-graph $\mathcal{H}$ (with $\pi(\mathcal{H})>0$ ). After this we form $\mathcal{F}^{*}$ by replacing any vertex from $\mathcal{F}$, except in Example 3, where we are more specific. Usually $\mathcal{H}$ is locally dense, and hence $\mathcal{F}$ is also locally dense. Consequently, Theorems 1 , 2 and (2) yield

$$
0<\pi(\mathcal{H})=\rho(\mathcal{H}) \leq \rho\left(\mathcal{F}^{*}\right)=\pi(\mathcal{F}) \leq \pi\left(\mathcal{F}^{*}\right)
$$

One only needs to verify that $\pi(\mathcal{F})<\pi\left(\mathcal{F}^{*}\right)$ to obtain $0<\rho\left(\mathcal{F}^{*}\right)<\pi\left(\mathcal{F}^{*}\right)$. Although this may be true in general, we are only able to show it for the few examples below.

Example 1. Let $H(4,3)$ be the (unique) four vertex triple system with three edges, and let $\mathcal{F}$ be a blowup of $H(4,3)$. Then Theorem 2 implies that $\rho\left(\mathcal{F}^{*}\right)=\pi(\mathcal{F})$. We also have $\pi(\mathcal{F})=\pi(H(4,3))$, and $2 / 7 \leq \pi(H(4,3))<1 / 3-10^{-6}$ (see $\left.[6,9]\right)$. Therefore

$$
2 / 7 \leq \rho\left(\mathcal{F}^{*}\right)<1 / 3-10^{-6}<4 / 9 \leq \pi\left(\mathcal{F}^{*}\right) .
$$

Note that in the case $\mathcal{F}=H(4,3)$, and the vertex used to form $\mathcal{F}^{*}$ is the unique vertex of degree three in $H(4,3)$, we obtain $\mathcal{F}^{*}=F(3,3)$, thus retrieving the example of [12].

Example 2. Let $F_{5}$ be the five vertex triple system with edges $123,124,345$. Let $\mathcal{F}$ be a blowup of $F_{5}$, where the vertex labelled 5 is replaced by at least two vertices. Then Theorem 2 implies that $\rho\left(\mathcal{F}^{*}\right)=\pi(\mathcal{F})$. We also have $\pi(\mathcal{F})=\pi\left(F_{5}\right)=2 / 9[5]$. Therefore

$$
2 / 9=\rho\left(\mathcal{F}^{*}\right)<4 / 9 \leq \pi\left(\mathcal{F}^{*}\right) .
$$

Example 3. Recall that $F(3,2)=\{567,467,367,345\}$. Let $\mathcal{F}$ be a blowup of $F(3,2)$. Let $\mathcal{F}^{*}$ be obtained from $\mathcal{F}$ by replacing one of the vertices playing the role of a vertex in $\{3,4,5\}$ (say 3 ), and then adding an edge among the three new vertices. Note that in the case $\mathcal{F}=F(3,2)$, we have $\mathcal{F}^{*}=\{567,467,367,345,267,167,245,145,123\}$, where 1 and 2 are the two new vertices, thus $\mathcal{F}^{*}=F_{7}$. Now Theorem 2 implies that $\rho\left(\mathcal{F}^{*}\right) \leq \pi(\mathcal{F})$. The last part of Theorem 2 implies that $\rho\left(\mathcal{F}^{*}\right)=\pi(\mathcal{F})$ except possibly in the case that $\mathcal{F}$ was obtained from $F(3,2)$ without blowing up any of the vertices $3,4,5$ (this includes the case $\mathcal{F}^{*}=F_{7}$ ).

In this case, let $F^{-}$be obtained from $\mathcal{F}^{*}$ by deleting the edge (in the labelling above) 123 . Then $F^{-}$is locally dense and so $\rho\left(F^{-}\right)=\pi\left(F^{-}\right)$by Theorem 1 . Consequently,

$$
4 / 9 \leq \pi(F(3,2))=\pi\left(F^{-}\right)=\rho\left(F^{-}\right) \leq \rho\left(\mathcal{F}^{*}\right) \leq \pi(\mathcal{F})=\pi(F(3,2)) \leq 4 / 9,
$$


where the first inequality is from [11], the first and last equalities are from (2), and the last inequality was recently proved by Füredi, Pikhurko, Simonovits [7]. Thus even in this case, $\rho\left(\mathcal{F}^{*}\right)=\pi(\mathcal{F})=$ $4 / 9$.

On the other hand, a short case analysis shows that $\mathcal{F}^{*}$ is absent in the hypergraph $\mathcal{G}$ with vertex partition $A_{1} \cup A_{2} \cup A_{3}\left(|| A_{i}|-| A_{j}|| \leq 1\right.$ for $\left.i \neq j\right)$, and all edges of the form $a b c$, where $a, b \in A_{i}, c \in A_{i+1}$ (indices modulo 3), or $a \in A_{1}, b \in A_{2}, c \in A_{3}$. Since $\mathcal{G}$ has density 5/9, $\pi\left(\mathcal{F}^{*}\right) \geq 5 / 9$. Therefore,

$$
4 / 9=\rho\left(\mathcal{F}^{*}\right)<5 / 9 \leq \pi\left(\mathcal{F}^{*}\right) .
$$

Example 4. Let $K_{4}^{3}$ be the complete triple system on four points, and let $\mathcal{F}$ be a blowup of $K_{4}^{3}$. Then Theorem 2 implies that $\rho\left(\mathcal{F}^{*}\right)=\pi(\mathcal{F})$. We also have $\pi(\mathcal{F})=\pi\left(K_{4}^{3}\right)$, and from $[2,17], 5 / 9 \leq$ $\pi\left(K_{4}^{3}\right)<.592$. It is easy to see that $\mathcal{F}^{*}$ is not 2-colorable, therefore $\pi\left(\mathcal{F}^{*}\right) \geq 3 / 4$. Consequently,

$$
5 / 9 \leq \rho\left(\mathcal{F}^{*}\right)<0.592<3 / 4 \leq \pi\left(\mathcal{F}^{*}\right)
$$

We end by remarking that our examples are nontrivial not only in the sense that the hypergraphs produced are irreducible, but also because one notes that $\rho$ is not preserved in general under the blowup operation (as $\pi$ is). Consequently, one cannot hope to just take blowups and produce an infinite family from a single 3-graph satisfying (1). One well-known problem in this regard is to determine $\rho\left(K_{2,2,2}\right)$, where $K_{2,2,2}$ is the complete 3 -partite graph with two vertices in each part. It is trivial that $\rho\left(K_{3}\right)=0$, but it is unknown whether $\rho\left(K_{2,2,2}\right)>0$.

\section{Acknowledgments}

These results were obtained when the first author visited the Rényi institute in March 2003. He thanks the Rényi institute for its support. He also thanks Vojta Rödl for discussions [13] that clarified the notion of irreducibility.

\section{References}

[1] B. Bollobás, P. Erdős, On a Ramsey-Turán type problem, J. Combinatorial Theory Ser. B 21 (1976), no. 2, 166-168. 
[2] F. R. K. Chung, L. Lu, An upper bound for the Turán number $t_{3}(n, 4)$, J. Combin. Theory Ser. A 87 (1999), no. 2, 381-389.

[3] P. Erdős, A. Hajnal, V. T. Sós, E. Szemerédi, More results on Ramsey-Turán type problems, Combinatorica 3 (1983), no. 1, 69-81.

[4] P. Erdős, V. T. Sós, On Ramsey-Turán type theorems for hypergraphs, Combinatorica 2 (1982), no. 3, 289-295.

[5] P. Frankl, Z. Füredi, A new generalization of the Erdős-Ko-Rado theorem, Combinatorica 3 (1983), no. 3-4, 341-349.

[6] P. Frankl, Z. Füredi, An exact result for 3-graphs. Discrete Math. 50 (1984), no. 2-3, 323-328.

[7] Z. Füredi, O. Pikhurko, M. Simonovits, On the Turán Density of the Hypergraph $\{a b c, a d e, b d e, c d e\}$, Electronic Journal of Combinatorics, 10 (2003), no. 1, Research Paper R18, 7 pp. (electronic).

[8] P. Frankl, V. Rödl, Some Ramsey-Turán type results for hypergraphs, Combinatorica 8 (1988), no. $4,323-332$.

[9] D. Mubayi, On hypergraphs with every four points spanning at most two triples, Electronic Journal of Combinatorics, 10 (2003), no. 1, Research Paper N10, 4 pp. (electronic).

[10] D. Mubayi, A hypergraph extension of Turán's theorem, to appear, J. Combin. Theory Ser. B

[11] D. Mubayi, V. Rödl, On the Turán number of triple systems, J. Combin Theory, Ser. A, 100 (2002), 136-152.

[12] D. Mubayi, V. Rödl, Supersaturation for Ramsey-Turán problems, to appear, Combinatorica.

[13] V. Rödl, personal communication, (2003).

[14] A. F. Sidorenko, On Ramsey-Turán numbers for 3-graphs, J. Graph Theory 16 (1992), no. 1, $73-78$.

[15] M. Simonovits, Extremal graph problems, degenerate extremal problems, and supersaturated graphs, Progress in graph theory (Waterloo, Ont., 1982), 419-437, Academic Press, Toronto, ON, 1984. 
[16] E. Szemerédi, On graphs containing no complete subgraph with 4 vertices, (Hungarian) Mat. Lapok 23 (1972), 113-116 (1973).

[17] P. Turán, Eine Extremalaufgabe aus der Graphentheorie, Mat. Fiz. Lapok 48 (1941) 436-452. 